\title{
High efficacy and safety of VTD as an induction protocol in patients with newly diagnosed multiple myeloma eligible for high dose therapy and autologous stem cell transplantation: A report of the Polish Myeloma Study Group
}

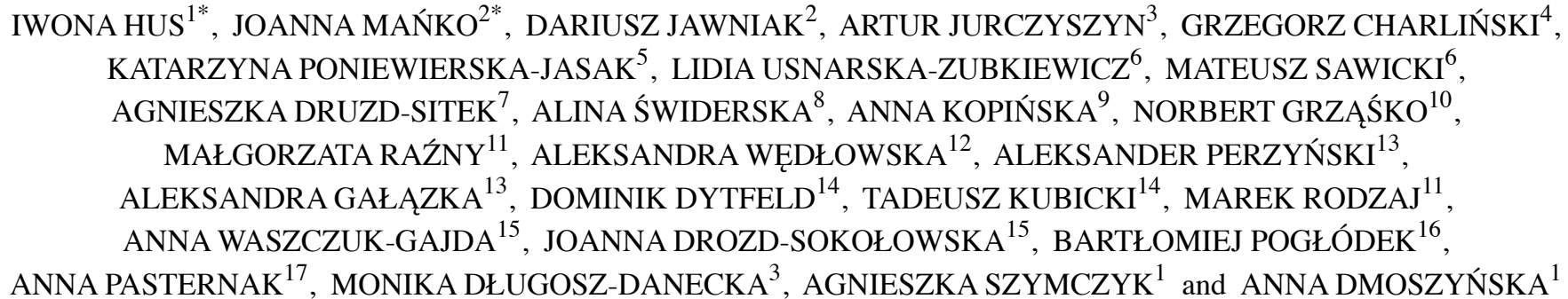

Departments of ${ }^{1}$ Clinical Transplantology, and ${ }^{2}$ Hematooncology and Bone Marrow Transplantation,

Medical University of Lublin, 20-081 Lublin; ${ }^{3}$ Department of Hematology, Collegium Medicum, Jagiellonian University, 30-051 Cracow; ${ }^{4}$ Department of Hematology, Specialist Muncipial Hospital, 87-100 Torun; ${ }^{5}$ Department of Hematology, Holycross Cancer Center, 25-734 Kielce; ${ }^{6}$ Department of Hematology, Blood Neoplasms and Bone Marrow Transplantation,

Wroclaw Medical University, 50-367 Wroclaw; ${ }^{7}$ Department of Lymphoid Malignancies, Institute of Oncology, 02-781 Warsaw; ${ }^{8}$ Department of Hematology, University Hospital in Zielona Góra, 65-046 Zielona Góra; ${ }^{9}$ Department of Hematology and Bone Marrow Transplantation, Medical University of Silesia, 40-032 Katowice; ${ }^{10}$ Department of Hematology, St. John of Dukla Lublin Region Cancer Center, 20-090 Lublin; ${ }^{11}$ Department of Hematology, Ludwik Rydygier Memorial Specialized Hospital, 31-826 Cracow; ${ }^{12}$ Department of Hematology and Transplantology, Medical University of Gdansk, 80-211 Gdansk; ${ }^{13}$ Department of Hematology, Institute of Hematology and Transfusion Medicine,

00-791 Warsaw; ${ }^{14}$ Department of Hematology and Bone Marrow Transplantation, Poznan University of Medical

Sciences, 60-569 Poznan; ${ }^{15}$ Department of Hematology, Oncology and Internal Medicine, Medical University of Warsaw, 02-097 Warsaw; ${ }^{16}$ Department of Oncologic Hematology, Specialist Hospital, 36-200 Brzozów; ${ }^{17}$ Department of Hematology, Independent Public Health Care of Ministry of The Interior and Administration, 10-228 Olsztyn, Poland

Received February 10, 2019; Accepted August 7, 2019

\section{DOI: $10.3892 / \mathrm{ol} .2019 .10929$}

\begin{abstract}
The present retrospective analysis evaluated the efficacy and safety of the VTD (bortezomib, thalidomide, dexamethasone) regimen in 205 newly-diagnosed patients with multiple myeloma (MM) eligible for high dose therapy and autologous stem cell transplantation (HDT/ASCT) in routine clinical practice. With a median of 6 cycles (range, 1-8), at least
\end{abstract}

Correspondence to: Dr Agnieszka Szymczyk, Department of Clinical Transplantology, Medical University of Lublin, Staszica Street 11, 20-081 Lublin, Poland

E-mail: agnieszka.szymczyk.med@wp.pl

*Contributed equally

Key words: multiple myeloma, first-line therapy, transplant-eligible patients, VTD partial response was achieved in $94.6 \%$ and at least very good partial response (VGPR) was achieved in $67.8 \%$ of patients. Peripheral neuropathy (PN) grade 2-4 was observed in $28.7 \%$ of patients. In $72 \%$ of patients undergoing stem cell mobilization one apheresis allowed the number of stem cells sufficient for transplantation to be obtained. Following HDT/ASCT the sCR rate increased from 4.9 to $14.4 \%$ and CR from 27.8 to $35.6 \%$. The results demonstrated that VTD as an induction regimen was highly efficient in transplant eligible patients with MM with increased at least VGPR rate following prolonged treatment ( $\geq 6$ cycles). Therapy exhibited no negative impact on stem cell collection, neutrophils and platelets engraftment following ASCT. Therapy was generally well tolerated and PN was the most common reason of dose reduction or treatment discontinuation.

\section{Introduction}

Multiple myeloma (MM) is a lymphoid malignancy characterized by clonal expansion of malignant plasma cells 
producing/secreting monoclonal protein (M-protein) as whole immunoglobulin molecules or light chain immunoglobulins only. Parameters correlating with negative outcome include IgA subtype of M-protein or light-chain multiple myeloma (LCMM), advanced disease stage according the International Staging System (ISS), high serum $\beta_{2}$-microglobulin and lactate dehydrogenase (LDH), low serum albumin and unfavorable cytogenetics, such as: $\mathrm{t}(4 ; 14), \mathrm{t}(14 ; 16)$, and del17p $(1,2)$. Treatment of MM has evolved during the last two decades which has resulted in unprecedented improvement of patients' outcome with increase of the median survival from 3 to 4 years to 7-8 years (3). This advancement started in the 1990s with the implementation of high-dose therapy followed by autologous stem cell transplantation (HDT/ASCT), however the most prominent effect for survival is associated with the introduction of novel targeted agents, including immunomodulatory drugs (IMIDs) and proteasome inhibitors (PIs) (4). The principles of MM first line therapy depend on the patients' eligibility for HDT/ASCT which is still recommended as a standard of care for patients younger than 65-70 years without prohibitive comorbidities that achieve at least a partial response for the induction therapy $(1,5)$. The efficacy of induction regimen is of key significance since the outcome after HDT/ASCT is highly related to the depth of response after induction therapy. Another important aspect is good tolerability and no negative impact on stem cell mobilization. Nowadays, three drug bortezomib-based regimens are generally recommended as a standard induction therapy for transplant-eligible patients with newly diagnosed MM (1,5-7). The choice between different regimens depends on drug availability in particular countries, their toxicity profile and local preferences. The objective of this retrospective analysis was to evaluate efficacy and safety of VTD regimen in newly diagnosed MM patients eligible for HDT/ASCT in routine clinical practice.

\section{Materials and methods}

Patients. In this retrospective analysis, we collected the data of transplant-eligible patients with measurable MM who received initial treatment with VTD regimen. Analytical work-flow taken by research group during the study is shown in Fig. 1.

The following inclusion criteria for the study were defined: Previously untreated, newly-diagnosed MM, age 18-70 years, ECOG performance status $<2$, patient's compliance with the given instructions, life expectancy $\geq 12$ months.

Key exclusion criteria were: Plasma cell leukaemia, light-chain amyloidosis, grade $\geq 2$ peripheral neuropathy (PN) or neuropathic pain, severe comorbidities, cardiac insufficiency (New York Heart Association (NYHA) >2 grade or left ventricular ejection fraction $<60 \%$, known human-immunodeficiency virus (HIV) infection, pregnant, breast-feeding or lactating women.

Treatment. VTD regimen recommended by the Polish Myeloma Study Group was as follows: Bortezomib at a dose of $1.3 \mathrm{mg} / \mathrm{m}^{2}$ administered subcutaneously (SC) or intravenous (IV) on days 1,4,8, and 11; dexamethasone at a dose $20-40 \mathrm{mg}$ on days 1-4 and 9-12; and thalidomide at a dose of 100-200 mg/day administered orally. Each cycle should be repeated every 21 days for up to 4-6 cycles (8). The analysis included medical records of patients who received at least one cycle of VTD. Concomitant medications included bisphosphonates, antiviral prophylaxis and/or antibiotics in accordance with local practice and deep venous thrombosis (DVT) prophylaxis with enoxaparin or acetylsalicylic acid (ASA). In patients achieving at least a partial response (PR) according to International Myeloma Working Group (IMWG) guidelines (9), peripheral blood hematopoietic stem cells (PBSC) were then mobilized according to the center's experience and practice. The minimal target yield was $2 \times 10^{6} \mathrm{CD} 34^{+}$cells $/ \mathrm{kg}$ for one transplant. The assumption was to obtain a sufficient number of $\mathrm{CD} 4^{+}$cells for two transplants. Following induction therapy and effective mobilization, patients proceeded to ASCT. The use of a conditioning regimen was left at the discretion of each center, as was the decision to conduct a single or tandem ASCT and the application of maintenance therapy. After transplantation, patients were followed every 12 weeks until disease progression, and then every 12 weeks for survival and subsequent therapies.

The response rates to the induction therapy as well as to the consolidation with HDT/ASCT, progression-free survival (PFS) and toxicity of the treatment were evaluated. Effectiveness of mobilization after VTD-induction therapy was also analyzed. The rresponse to the therapy was evaluated according to the updated IMWG uniform response criteria (9). Adverse events (AEs) were graded by the Common Terminology Criteria for Adverse Events, version 4.03. [http://evs.nci.nih. gov/ftp1/CTCAE/CTCAE_4.03_2010-06-14_QuickReference_5x7. pdf].

Statistical analysis. The influence of categorized parameters on the response to the treatment and toxicity was performed using the Pearson chi square test and exact Fisher test. Associations between response and patient characteristics for continuous parameters were evaluated using the Mann-Whitney U test. Survival curves were estimated by the Kaplan-Meier method and the log-rank test was applied for comparison. PFS was defined as the time from introducing the treatment to the last date on which the disease activity was assessed, including death from any reason. Follow-up time was short to achieve reliable results concerning overall survival. The influence of independent variables on patient survival was tested by univariate and multivariate Cox proportional hazards regression. A multi-parameter analysis of factors predicting response to the treatment was also performed.

\section{Results}

Patients. Between June 2015 and June 2017, a cohort of 205 consecutive patients qualified for HDT/ASCT were treated with VTD as induction therapy in 17 Polish hematology/oncology centers. The median age of patients was 59 years (range: 34-70 years). Advanced clinical stage according ISS (International Staging System) (10) was found in 88 patients (43\%). R-ISS (11) was evaluated in only in 90 patients and stage III was found in 20 patients $(22.2 \%)$. Non-IgG type of M-protein was found in 83 patients (40.5\%). Cytogenetics was evaluated by FISH in 100 patients, with abnormalities found in 38 patients (38\%), including 20 patients with adverse prognostic lesions, such as: $\mathrm{t}(4 ; 14), \mathrm{t}(11 ; 14)$ and abnormalities of 17 chromosome. Polyneuropathy was present in 4 patients (1.9\%) before 


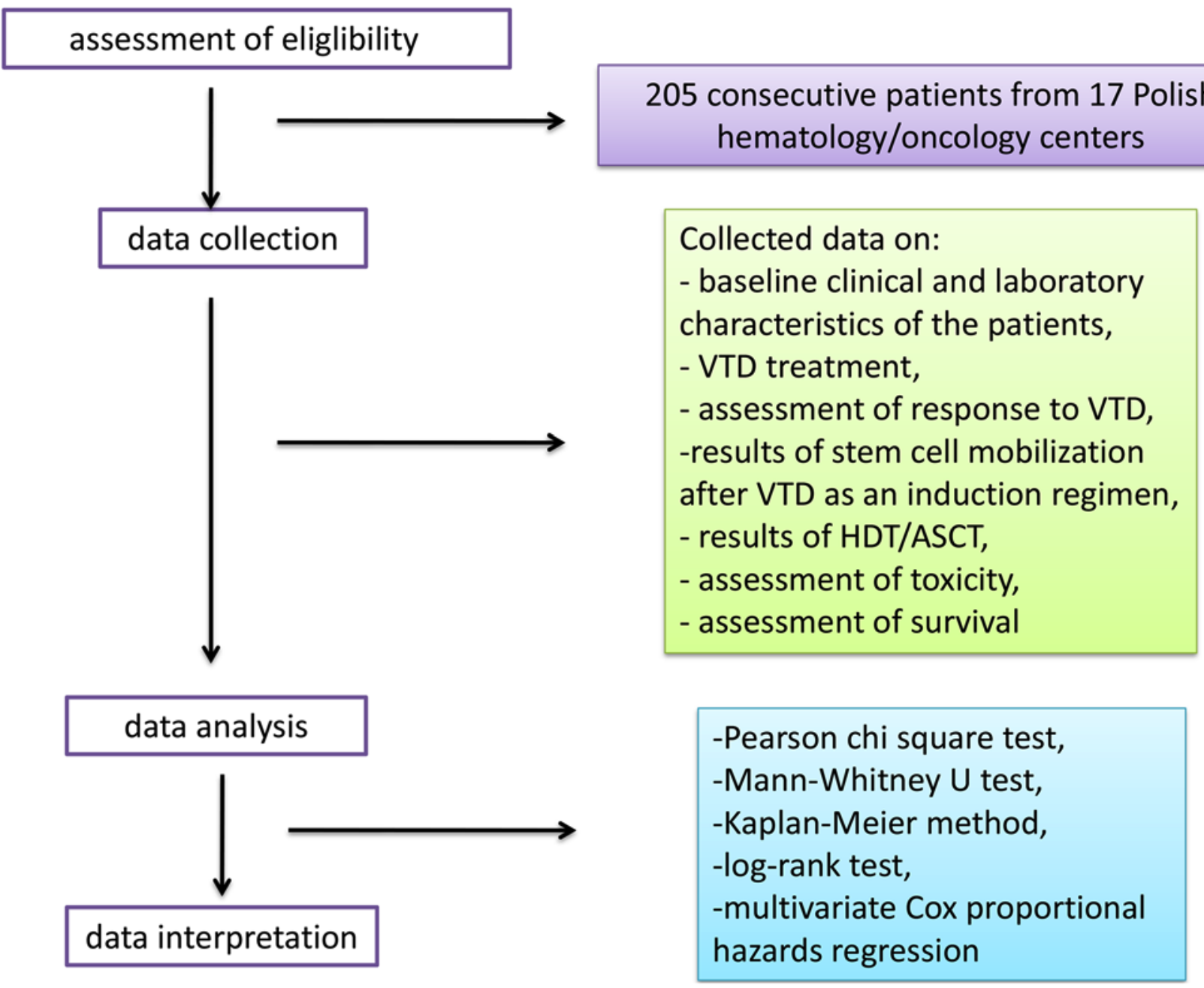

Figure 1. Analytical work-flow of the present retrospective study. HDT/ASCT, high-dose therapy followed by autologous stem cell transplantation; VTD, bortezomib, thalidomide, dexamethasone.

the start of VTD therapy. Baseline clinical and laboratory characteristics of the patients are outlined in Table I.

Treatment. The data on the drug dosage were available for 184 patients $(90 \%)$. All the patients started the therapy with the standard dose of bortezomib $\left(1.3 \mathrm{mg} / \mathrm{m}^{2}\right.$, in days $\left.1,4,8,11\right)$. Bortezomib was administered SC in 154 patients (75.1\%), IV in 41 patients $(20 \%)$ and 10 patients received bortezomib both routes. In 170 patients $(92.9 \%)$, thalidomide was administered in a dose of $100 \mathrm{mg} / \mathrm{d}$ and in 11 patients in the dose of $200 \mathrm{mg} / \mathrm{d}$. In 91 patients $(44.3 \%)$ the dose of dexamethasone was $160 \mathrm{mg} / \mathrm{cycle}$, and in 90 patients (43.9\%)-320 mg/cycle. In 90 patients (43.9\%) VTD was given in 21-day cycles and in 115 patients in 28-day cycles (56\%). During therapy, all patients received anti-DVT prophylaxis. LMWH (low-molecular weight heparin) was used in 119 patients $(58 \%)$ and ASA was used in 70 patients $(34.1 \%)$. Antiviral prophylaxis with acyclovir was used routinely in all of patients. The median number of cycles was 6 (range 1-8), 43 patients received 3-4 cycles (21\%), 107 patients received 5-6 cycles $(52 \%)$ and 47 patients received 7-8 cycles $(23 \%)$.

Assessment of response. All 205 patients were available for the evaluation of response. ORR was $94.6 \%$, $(n=194)$ including $32.7 \%$ of $\geq \mathrm{CR}$ and $67.8 \%$ of $\geq \mathrm{VGPR}$. sCR was achieved in $4.9 \%$ of patients, $(n=10)$, CR in $27.8 \%(n=57)$, VGPR in $35.1 \%(n=72)$ and $\mathrm{PR}$ in $27.3 \%$ of patients $(\mathrm{n}=56)$. Stable disease was observed in $2.4 \%$ of patients $(n=5)$ and disease progression in $2.4 \%$ of patients $(n=5)$. In patients with high-risk cytogenetics there was a lower rate of $\mathrm{CR}$ and $\mathrm{SCR}$ as compared to the group without these abnormalities (23.8\% vs. 30.4 and $0 \%$ vs. $10.1 \%$ ), but not statistically significant $(\mathrm{P}>0.05)$. There was no significant relationship between pre-treatment laboratory parameters, steroids dose (160 vs. $320 \mathrm{mg}$ ), thalidomide dose (200 vs. $100 \mathrm{mg}$ ), bortezomib dose and route of administration and the achievement of response for the therapy $(\mathrm{P}>0.05)$. Response rates were higher after $\geq 6$ cycles of VTD as compared to the response rates after the 4th cycle, but the difference was not statistically significant (P>0.05; Fig. 2).

Assessment of survival. After the median follow-up of 18 months, PFS was $83.1 \%$. The only parameter associated with longer PFS was the number of administered VTD cycles $>4$ $(\mathrm{P}<0.05$; Fig. 3). In patients with renal failure PFS was similar to the patients with normal renal function.

Toxicity. The most common non-hematological grade adverse event was PN that occurred in $44.5 \%$ of patients (Table II). The incidence of $\mathrm{PN}$ was similar in patients receiving bortezomib IV 
Table I. Clinical characteristics of patients treated with the VTD regimen.

\begin{tabular}{|c|c|}
\hline Parameter & Value \\
\hline \multicolumn{2}{|l|}{ Sex, n (\%) } \\
\hline Female & $97(47.3)$ \\
\hline Male & $108(52.7)$ \\
\hline \multicolumn{2}{|l|}{ ISS stage ${ }^{\mathrm{a}}, \mathrm{n}(\%)$} \\
\hline 1 & $71(34.6)$ \\
\hline 2 & $45(22.4)$ \\
\hline 3 & $88(43.0)$ \\
\hline \multicolumn{2}{|l|}{ R-ISS stage ${ }^{\mathrm{b}}, \mathrm{n}(\%)$} \\
\hline 1 & $22(24.4)$ \\
\hline 2 & $47(52.2)$ \\
\hline 3 & $20(22.2)$ \\
\hline \multicolumn{2}{|l|}{ Protein M type, n (\%) } \\
\hline $\operatorname{IgG}$ & $118(57.6)$ \\
\hline $\operatorname{Ig} \mathrm{A}$ & $41(20.0)$ \\
\hline Light chain MM & $39(19.0)$ \\
\hline $\operatorname{IgM}$ & $3(1.45)$ \\
\hline \multicolumn{2}{|l|}{ Monoclonal light chain, n (\%) } \\
\hline Kappa & $113(55.1)$ \\
\hline Lambda & $88(42.9)$ \\
\hline Non-secretory & $4(1.95)$ \\
\hline Albumin, g/l, median; (min-max) & $3.6 ;(1.8-5.2)$ \\
\hline $\begin{array}{l}\beta \text {-2-microglobulin, } \mathrm{mg} / \mathrm{l} \\
\text { median; (min-max) }\end{array}$ & $4.1 ;(1.58-60.0)$ \\
\hline Creatinine, $\mathrm{mg} / \mathrm{dl}$, median; (min-max) & $0.92 ;(0.37-10.57)$ \\
\hline $\begin{array}{l}\text { GFR acc. MDRD formula, } \mathrm{ml} / \mathrm{min} \text {, } \\
\text { median; (min-max) }\end{array}$ & $60 ;(53-150)$ \\
\hline $\begin{array}{l}\text { Hemoglobin, g/dl, median; } \\
(\min -\max )\end{array}$ & $10.9 ;(6.0-17.3)$ \\
\hline Neutrophils, G/1, median; (min-max) & $3.2 ;(0.91-10.1)$ \\
\hline Platelets, G/1, median; (min-max) & $206 ;(54-552)$ \\
\hline $\begin{array}{l}\text { Calcium, mmol/1, } \\
\text { median; (min-max) }\end{array}$ & $2.39 ;(1.8-4.77)$ \\
\hline $\begin{array}{l}\text { Monoclonal protein, } \mathrm{g} / \mathrm{dl} \text {, } \\
\text { median; (min-max) }\end{array}$ & $3.59 ;(0-14.5)$ \\
\hline $\begin{array}{l}\text { Plasma cells, bone marrow, } \\
\% \text { median; }(\min -\max )\end{array}$ & $37 ;(0-95)$ \\
\hline Cytogenetics, del 17p, t(4;14) $\mathrm{t}(14 ; 16)$ & $21 / 100$ \\
\hline
\end{tabular}

${ }^{\text {aISS }}$ was evaluated in 204 patients, for one patient data were unavailable. ${ }^{b} \mathrm{R}$-ISS was evaluated in 89 patients (43.4\%), since cytogenetic assessment is not a standard procedure in all hematological centers in Poland. ISS, International Staging System; R-ISS, revised International Staging System; VTD, bortezomib, thalidomide, dexamethasone.

(39\%-all grades and $2.44 \%$-severe) and SC (45.5\%-all grades and $4.55 \%$-severe). VZV (varicella zoster virus) reactivation was observed in $3.9 \%(8 / 205)$ of patients. The rates of hematological adverse events are presented in Table III.

Bortezomib dose was reduced in 42 patients (20.5\%). PN was the main reason of bortezomib dose reduction (33 patients; 78.6\%;

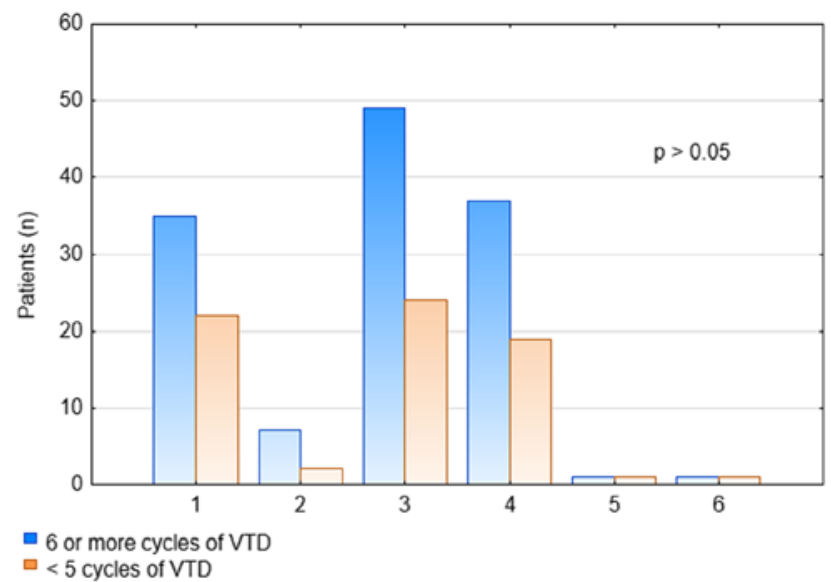

Figure 2. Association between number of administered VTD cycles and response rates. VTD, bortezomib, thalidomide, dexamethasone.

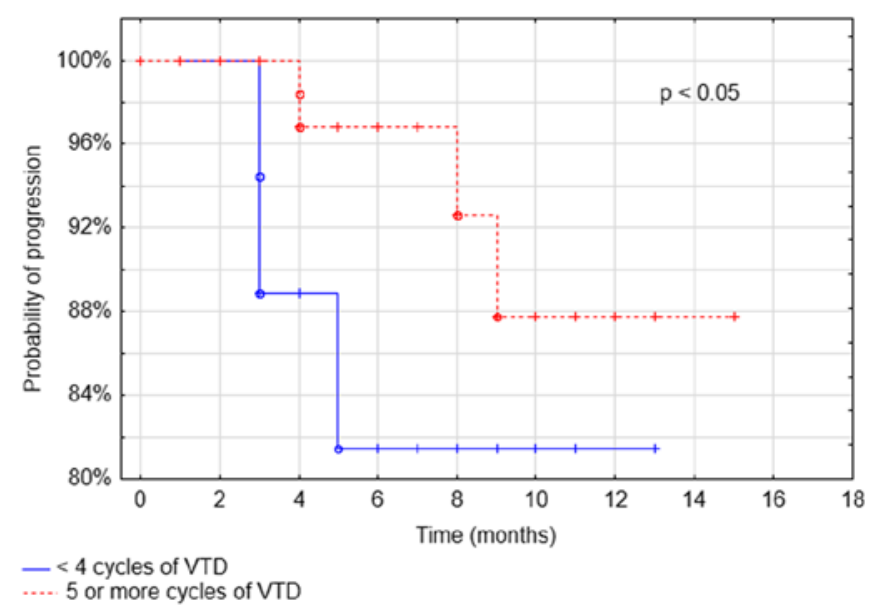

Figure 3. Association between progression-free survival and number of administered VTD cycles. VTD, bortezomib, thalidomide, dexamethasone.

grade 2 neuropathy with pain-23 patients, grade 3-10 patients), among the other reasons there were: Skin changes in 2 patients (4.7\%), lung fibrosis in 2 patients (4.7\%). Severe thrombocytopenia and neutropenia were the reason of dose reduction only in 1 patient each. Bortezomib treatment was discontinued in 26 patients $(12.7 \%)$. The main reasons for discontinuation were adverse events (88.4\%), including PN in 9 patients (34.6\%), thrombotic complications (thrombosis, pulmonary embolism) in 5 patients $(19.2 \%)$. Thalidomide treatment was discontinued in 3 patient (1.5\%) due to polyneuropathy. In $8(3.9 \%)$ patients with grade 3 polyneuropathy and $3(1.5 \%)$ patients with grade 2 polyneuropathy, the dose of thalidomide was reduced to $50 \mathrm{mg}$.

On the basis of the exact Fisher test, it was found that there was no significant relationship between the dose of steroids (160 vs. $320 \mathrm{mg}$ ), the dose of thalidomide ( $200 \mathrm{vs} .100 \mathrm{mg}$ ), route of bortezomib administration (SC vs.IV), duration of cycle (21 days vs. 28 days), and toxicity of VTD therapy ( $>0.05$ ). However, it was shown that in the group of patients in whom the dose of bortezomib was reduced, polyneuropathy was statistically significantly more frequent $(\mathrm{P}<0.05$; Table IV). No correlation between the number of treatment cycles, dose of thalidomide and occurrence of polyneuropathy and other adverse events 
Table II. Non-hematological toxicity of the VTD regimen.

\begin{tabular}{|c|c|c|c|c|}
\hline \multirow{2}{*}{$\begin{array}{l}\text { Non-hematological } \\
\text { toxicity }\end{array}$} & \multicolumn{2}{|c|}{ 1-2 grade adverse events } & \multicolumn{2}{|c|}{ 3-4 grade adverse events } \\
\hline & Number, $\mathrm{n}$ & Percentage $(n=205)$ & Number, $\mathrm{n}$ & Percentage $(n=205)$ \\
\hline \multicolumn{5}{|l|}{ Polyneuropathy } \\
\hline Grade 1 & 36 & 17.6 & 10 & 4.9 \\
\hline Grade 2 & 45 & 33.0 & & \\
\hline Infections & 8 & 4.0 & 2 & 1.0 \\
\hline Thrombosis & 7 & 3.4 & 1 & 0.5 \\
\hline Pulmonary embolism & - & - & 2 & 1.0 \\
\hline Constipation & 2 & 1.0 & - & - \\
\hline Skin alterations & 2 & 1.0 & 3 & 1.5 \\
\hline
\end{tabular}

VTD, bortezomib, thalidomide, dexamethasone.

Table III. Hematological toxicity of VTD regimen.

\begin{tabular}{|c|c|c|c|c|}
\hline \multirow[b]{2}{*}{ Hematological toxicity } & \multicolumn{2}{|c|}{ 1-2 grade adverse events } & \multicolumn{2}{|c|}{ 3-4 grade adverse events } \\
\hline & Number, $\mathrm{n}$ & Percentage $(n=205)$ & Number, $\mathrm{n}$ & Percentage $(n=205)$ \\
\hline Neutropenia & 1 & 0.5 & 5 & 2.5 \\
\hline Thrombocytopenia & 4 & 2.0 & 1 & 0.5 \\
\hline Anemia & 12 & 6.0 & - & - \\
\hline
\end{tabular}

VTD, bortezomib, thalidomide, dexamethasone.

( $\mathrm{P}>0.05$; Table IV). In 3 patients, treatment was discontinued because of the lack of efficacy. Six patients died during the median 8.56 months follow-up (range 2.8-24.9). There were three early deaths and the reasons were sepsis ( 2 patients after 2 and 3 cycles of VTD, respectively) and heart failure ( 1 patient after 6 cycles of VTD). One patient died from pneumonia before $\mathrm{CD} 34^{+}$cell mobilization, one patient from sepsis after ASCT (+59 day) and one due to MM progression (19 months after VTD completion).

Results of stem cell mobilization after VTD as an induction regimen. Hematopoietic stem cell mobilization was performed at the time of analysis in 146 patients (71\%). In $63.7 \%$ of patients $(n=93)$ one apheresis allowed the number of stem cells sufficient for transplantation to be obtained. In $20 \%$ of patients $(n=29)$ two apheresis were performed. The median yield of $\mathrm{CD} 34^{+}$cells was $12.6 \times 10^{6} / \mathrm{kg}\left(\max 70 \times 10^{6} / \mathrm{kg}\right)$ which was sufficient for two transplantations in the majority of patients.

Most commonly used protocols before HSCT mobilization were cytosine arabinoside $(n=53,36.3 \%)$ and cyclophosphamide $(n=63 ; 43.1 \%)$. The other protocols included: Etoposide $(n=11$; 7.5\%); G-CSF alone ( $\mathrm{n}=11 ; 7.5 \%)$; cyclophosphamide with etoposide $(n=7 ; 4.8 \%)$ and plerixafor $(n=1 ; 0.8 \%)$. The median yield of $\mathrm{CD} 34^{+}$cells was the highest in patients mobilized with cytosine arabinoside. Protocols used before HSCT mobilization and their efficacy are presented in Table V. In three patients that needed a second procedure of stem cell mobilization, protocols with cytosine arabinoside, G-CSF and plerixafor were used and the number of HSCT sufficient for the transplant was obtained (median 3.56x10\% $\mathrm{kg}$; range 3.02-4.1) Table V.

Results of HDT/ASCT after VTD as an induction regimen. HDT/ASCT was performed at the time of analysis in 128 patients with MEL 200 (melphalan $200 \mathrm{mg} / \mathrm{m}^{2}$ ) protocol as conditioning regimen in $87.5 \%$ of patients. The other conditioning protocols included: MEL 140 (8.6\%) and TMI (total marrow irradiation) with $12 \mathrm{~Gy}(2.3 \%)$; treosulfan+MEL $(0.8 \%)$; TBI+MEL $(0.8 \%)$.

The median number of transplanted $\mathrm{CD} 34^{+}$cells was $5.4 \times 10^{6} / \mathrm{kg}$ (range: $3.1-10.6 \times 10^{6} / \mathrm{kg}$ ). The median time to reach ANC (absolute neutrophil count) $>0.5 \mathrm{G} / 1$ and PLT (platelets) count $>20 \mathrm{G} / 1$ were 11 and 12 days, respectively. The most common grade $\geq 3$ adverse events observed after HDT/ASCT were infections noted in 28 patients $(21.9 \%)$, including gastrointestinal tract infections in 9 patients $(7 \%)$, mucositis in 7 patients $(5.5 \%)$, neutropenic fever in 7 patients $(5.5 \%)$ and sepsis in 3 patients $(2.3 \%)$. CMV (cytomegalovirus) infection was observed in 2 patients (1.6\%).

Evaluation of response 100 days after HDT/ASCT was performed in 104 patients. Comparing the response rates after HDT/ASCT to the responses after the induction, there was an increase of $\geq \mathrm{CR}(50 \%$ vs. $32.7 \%)$ and $\geq \operatorname{VGPR}(85.6 \%$ vs. $67.8 \%$ ). CR rate increased from 27 to $35.6 \%$ and sCR rate increased from 4.9 to $14.4 \%$ (Fig. 4). 
Table IV. Assessment of non-hematological and hematological adverse events incidence during VTD therapy.

\begin{tabular}{|c|c|c|c|}
\hline \multirow[b]{2}{*}{ Analyzed parameter } & \multicolumn{2}{|c|}{ Occurrence } & \multirow[b]{2}{*}{ P-value } \\
\hline & Yes $(\%)$ & No $(\%)$ & \\
\hline
\end{tabular}

Polyneuropathy

Number of VTD cycles

$$
\geq 6
$$$$
<5
$$

Duration of cycle

$$
21 \text { days }
$$

28 days

Initial dose of thalidomide

$$
\begin{aligned}
& 200 \mathrm{mg} \\
& 100 \mathrm{mg}
\end{aligned}
$$$$
<100 \mathrm{mg}
$$

Bortezomib dose reduction

Yes

No

Infection

Number of VTD cycles

$\geq 6$
$<5$

Duration of cycle

21 days

28 days

Initial dose of thalidomide

$200 \mathrm{mg}$

$100 \mathrm{mg}$

$<100 \mathrm{mg}$

Bortezomib dose reduction

Yes

No

Thrombosis

Number of VTD cycles

$$
\geq 6
$$

$<5$

Duration of cycle

$$
21 \text { days }
$$

28 days

Initial dose of thalidomide

$$
200 \mathrm{mg}
$$

$100 \mathrm{mg}$

$<100 \mathrm{mg}$

Bortezomib dose reduction

Yes

No

Pulmonary embolism

Number of VTD cycles

$$
\geq 6
$$$$
<5
$$

$5.5>0.05$

0.6

1.6

0.0

$62.4>0.05$

33.7

62.4

32.7

$41.0>0.05$

54.1

$11.0>0.05$

165

2.0

$18.5>0.05$

76.6

$41.9>0.05$

54.1

91.2

1.1

\begin{tabular}{|c|c|c|c|}
\hline \multirow[b]{2}{*}{ Analyzed parameter } & \multicolumn{2}{|c|}{ Occurrence } & \multirow[b]{2}{*}{ P-value } \\
\hline & Yes $(\%)$ & No $(\%)$ & \\
\hline \multicolumn{4}{|c|}{ Initial dose of thalidomide } \\
\hline $200 \mathrm{mg}$ & 0.0 & 6.0 & $>0.05$ \\
\hline $100 \mathrm{mg}$ & 1.1 & 91.8 & \\
\hline$<100 \mathrm{mg}$ & 0.0 & 1.1 & \\
\hline \multicolumn{4}{|c|}{ Bortezomib dose reduction } \\
\hline Yes & 0.0 & 20.5 & $>0.05$ \\
\hline No & 1.0 & 78.5 & \\
\hline \multicolumn{4}{|l|}{ Constipations } \\
\hline \multicolumn{4}{|c|}{ Number of VTD cycles } \\
\hline$\geq 6$ & 1.0 & 64.4 & $>0.05$ \\
\hline$<5$ & 0.0 & 34.6 & \\
\hline \multicolumn{4}{|l|}{ Duration of cycle } \\
\hline 21 days & 0.0 & 43.9 & $>0.05$ \\
\hline 28 days & 1.0 & 55.1 & \\
\hline \multicolumn{4}{|c|}{ Initial dose of thalidomide } \\
\hline $200 \mathrm{mg}$ & 0.0 & 6.0 & $>0.05$ \\
\hline $100 \mathrm{mg}$ & 0.0 & 92.9 & \\
\hline$<100 \mathrm{mg}$ & 0.0 & 1.1 & \\
\hline
\end{tabular}

$19.0>0.05$

77.1

0.0

21 days

28 days
1.0
Table IV. Continued.

Bortezomib dose reduction Yes

$\begin{array}{lll}0.0 & 20.5 & >0.05\end{array}$

$$
\text { No }
$$

$1.0 \quad 79.5$

Skin alterations

Number of VTD cycles

$\geq 6$

$<5$

1.0

1.5

$64.4>0.05$

Duration of cycle

$$
21 \text { days }
$$

28 days

$\begin{array}{lll}1.0 & 38.1 & >0.05\end{array}$

$1.5 \quad 54.6$

Initial dose of thalidomide

$$
200 \mathrm{mg}
$$

$100 \mathrm{mg}$

1.1

4.8

$<100 \mathrm{mg}$

0.5

91.6

$0.0 \quad 0.0$

Bortezomib dose reduction

$$
\text { Yes }
$$

0.5

20.0

$>0.05$

No

$2.0 \quad 77.5$

Neutropenia

Number of VTD cycles

$\geq 6$

$\begin{array}{lll}2.4 & 63.0 & >0.05\end{array}$

$<5$

$0.5 \quad 34.1$

Duration of cycle

21 days

1.0

$42.9>0.05$

28 days

2.0

54.1

Initial dose of thalidomide

$$
200 \mathrm{mg}
$$

$100 \mathrm{mg}$

0.55

5.5

$>0.05$

$<100 \mathrm{mg}$

$0.55 \quad 92.3$

$0.0 \quad 1.1$

Bortezomib dose reduction

Yes

$0.0 \quad 20.5$

$>0.05$ 
Table IV. Continued.

\begin{tabular}{|c|c|c|c|}
\hline \multirow[b]{2}{*}{ Analyzed parameter } & \multicolumn{2}{|c|}{ Occurrence } & \multirow[b]{2}{*}{ P-value } \\
\hline & Yes $(\%)$ & No $(\%)$ & \\
\hline \multicolumn{4}{|l|}{ Thrombocytopenia } \\
\hline \multicolumn{4}{|c|}{ Number of VTD cycles } \\
\hline$\geq 6$ & 0.5 & 64.9 & $>0.05$ \\
\hline$<5$ & 1.5 & 33.2 & \\
\hline \multicolumn{4}{|l|}{ Duration of cycle } \\
\hline 21 days & 2.0 & 41.9 & $>0.05$ \\
\hline 28 days & 0.0 & 56.1 & \\
\hline \multicolumn{4}{|c|}{ Initial dose of thalidomide } \\
\hline $200 \mathrm{mg}$ & 1.1 & 4.9 & $>0.05$ \\
\hline $100 \mathrm{mg}$ & 0.6 & 92.3 & \\
\hline$<100 \mathrm{mg}$ & 0.0 & 1.1 & \\
\hline \multicolumn{4}{|c|}{ Bortezomib dose reduction } \\
\hline Yes & 0.5 & 20.0 & $>0.05$ \\
\hline No & 1.5 & 78.0 & \\
\hline \multicolumn{4}{|l|}{ Anemia } \\
\hline \multicolumn{4}{|c|}{ Number of VTD cycles } \\
\hline$\geq 6$ & 2.9 & 62.4 & $>0.05$ \\
\hline$<5$ & 2.9 & 31.8 & \\
\hline \multicolumn{4}{|l|}{ Duration of cycle } \\
\hline 21 days & 4.4 & 39.5 & $>0.05$ \\
\hline 28 days & 1.5 & 54.6 & \\
\hline \multicolumn{4}{|c|}{ Initial dose of thalidomide } \\
\hline $200 \mathrm{mg}$ & 3.3 & 2.7 & $>0.05$ \\
\hline $100 \mathrm{mg}$ & 1.1 & 91.8 & \\
\hline$<100 \mathrm{mg}$ & 0.0 & 1.1 & \\
\hline \multicolumn{4}{|c|}{ Bortezomib dose reduction } \\
\hline Yes & 1.5 & 19.0 & $>0.05$ \\
\hline No & 4.4 & 75.1 & \\
\hline
\end{tabular}

VTD, bortezomib, thalidomide, dexamethasone.

Tandem HDT/ASCT was performed in 21 patients with suboptimal response to the first course of HDT. Conditioning protocols included: MEL200 in 15 patients and MEL140 in 4 patients, TMI in 2 patients, achieving significant improvement in the quality of response (Pearson test, $\mathrm{P}<0.001$ ) with increase of CR to $52.4 \%$ (11 patients).

\section{Discussion}

In the era of modern therapies, consolidation with HDT/ASCT is still considered as a standard of first line treatment in eligible MM patients since evidence from phase 3 studies demonstrated the improvement in the depth of response and progression-free survival $(12,13)$. Three-drug regimens containing PI and IMID were shown to be superior than three-drug regimens with either PI or IMID as well as than two-drug regimens in the terms of response rates and survival (14-20). Currently in Poland, VTD is the most common induction regimen used in about $85 \%$ of patients, while VCD being used only in the minority of them.
Table V. Efficacy of the protocols used prior to HSCT mobilization.

\begin{tabular}{lccc}
\hline & \multicolumn{3}{c}{ Yield of CD34 ${ }^{+}$cells $\left(x 10^{6} / \mathrm{kg}\right)$} \\
\cline { 2 - 4 } Mobilization protocol & Median & Minimum & Maximum \\
\hline CPX+G-CSF & 9.9 & 3.1 & 30.0 \\
VEP +G-CSF & 18.0 & 6.5 & 55.0 \\
ID-AraC+G-CSF & 20.0 & 3.1 & 70.0 \\
G-CSF alone & 7.2 & 4.6 & 17.0
\end{tabular}

CPX, cyclophosphamide; VEP, etoposide; ID-AraC, cytosine arabinoside; G-CSF, granulocyte-colony stimulating factor; HSCT, hematopoietic stem cell transplantation.

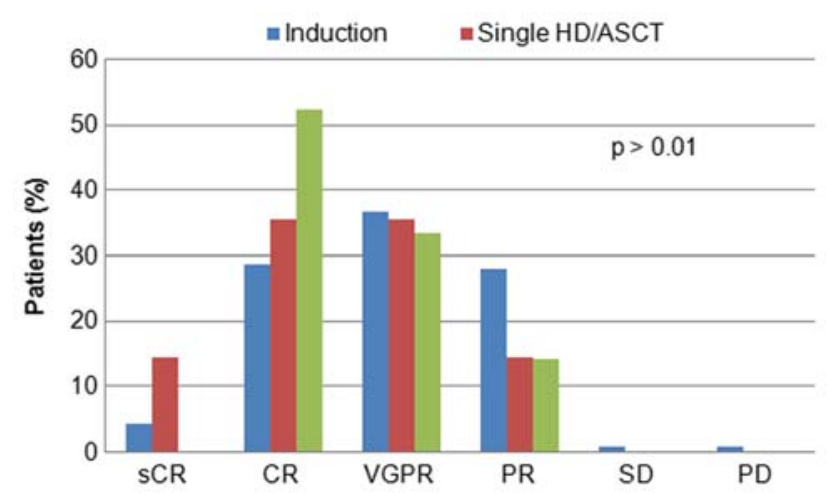

Figure 4. Evaluation of response following induction therapy, HDT/ASCT and double HDT/ASCT. HDT/ASCT, high-dose therapy followed by autologous stem cell transplantation; PR, partial response; VGPR, very good partial response; sCR, stringent complete remission; $\mathrm{CR}$, complete remission; $\mathrm{SD}$, stable disease; $\mathrm{PD}$, progressive disease.

Our data show that high response rates with $\geq$ VGPR rate of $67.8 \%$ could be achieved with VTD in clinical practice being in line with the data from clinical trials where at least VGPR ranged from 49 to $69 \%(14-16,21,22)$.

However, in phase 3 clinical trial IFM2013-04, at least VGPR was achieved in $66.3 \%$ of patients after 4 cycles of VTD (16) and in retrospective case-matched analysis by Cavo et al (23). in $64 \%$ of patients treated with 3 cycles of VTD regimen. The median number of cycles in our study was 6 , so, in clinical practice a higher number of VTD cycles was needed to achieve results comparable to the clinical studies. There are a few possible explanations of these discrepancies. First, there were differences in study groups characteristics, like, for example a higher percentage of the patients with a more advanced stage of MM in the group in our study as compared to the IFM2013-04 trial. What would be even more important, in our retrospective analysis, cytogenetics profile was unknown in about half of the patients and as it demonstrated in so far published data, bortezomib could only partially overcome the adverse prognostic impact associated with high risk cytogenetics. The results of the IFM group study showed that it could eliminate the poor risk prognosis of $t(4 ; 14)(24)$, however the data concerning $\operatorname{del}(17 \mathrm{p})$ remain unsatisfactory $(14,22,25-29)$. Another reason might be probably the lower median dose intensity caused by 
more arbitrary approach to dose reduction and discontinuation than in clinical trials. The rate of $\geq \mathrm{CR}$ after the induction with VTD in our study was $32.7 \%$ that was much higher than in the IFM2013-04 trial (16) and Italian case-matchedanalysis (23). It was comparable though to the results obtained in the Spanish PETHEMA Group study after 6 cycles of VDT (22), where a significant proportion of patients achieved CR during the three final cycles. As it was demonstrated by the results of IFM 2005-01 trial, achieving at least VGPR before transplant was associated with longer PFS (30). So, it might be useful continuing the therapy to increase the quality of response especially in patients with adverse cytogenetics or in patients in which cytogenetics is unknown, like it happens in clinical practice, since achievement of less than VGPR was a stronger predictor for progression than cytogenetics (30). According to the most recent ESMO guidelines 4 to 6 cycles of induction regimen is recommended before HDT/ASCT (1). As much as $23 \%$ of patients in our retrospective analysis received more than 6 cycles of VTD. As we think, this could have been related to non-medical conditions, like delayed patients' decision on undergoing HDT/ASCT or extended time of waiting for transplantation procedure. Lower doses of dexamethasone (160 vs. $320 \mathrm{mg}$ ) as well as thalidomide (100 vs. $200 \mathrm{mg}$ ) were as effective as the higher ones confirming once again that lower, less toxic doses should be preferred (31).

Among the regimens used in induction treatment in MM patients eligible for HDT/ASCT, VRD (bortezomib, lenalidomid, dexamethasone) is considered as very effective both in the context of response rates (32), as well as the benefit in OS (VRd vs. Rd) that was not was observed in case of VTD (17). However, lenalidomide is not yet approved in the first-line therapy of patients eligible to HDT/ASCT and the much higher cost of VRD compared to VTD would be an issue in some countries.

In this retrospective analysis, PN grade 2 was reported in $22 \%$ of patients and grade 3 in $4.9 \%$ of patients even though the median number of cycles was 6 . Therefore, grade 2-4 PN (26.9\%) was lower than expected basing on the results of clinical trials $(16,33,34)$. The possible explanation is that in clinical practice the grading of neuropathy was determined by the physicians caring for these patients. Presumably hematologists tend to minimize or 'down grade' these complaints, especially in patients that benefit from the therapy. Given the subjective nature of grading these symptoms, it would perhaps more helpful to look at the number of patients who had to be either dose reduced or who discontinued therapy due to neuropathy (20.5\%). This means that twice weekly bortezomib (given IV or SC) in combination with thalidomide/dexamethasone leads to significant neuropathy. However, PN was the reason for bortezomib discontinuation only in 9/205 patients (4.4\%). Thalidomide dose was reduced even in fewer patients, $(8 / 3.9 \%)$, though according to the guidelines, the dose of thalidomide should be reduced first due to its irreversibility. It is possible that the dose of $100 \mathrm{mg}$ was presumed to be low by the treating physicians and that is why they reduced bortezomib first. These observations suggest that vigilance for neuropathy should be higher in clinical practice since along with prolongation of patients survival its negative influence of quality of life is extremely important. It would be advisable to include independent neurological assessment in patients' management. While lenalidomide may not be available for first line therapy an easy, less toxic (and perhaps more effective) solution may be weekly bortezomib which has been shown to lead to less neuropathy and could be potential option to improve tolerability.

In contrast to the results of the phase 3 clinical trial (35), but similar to our previous retrospective analysis on VMP in clinical practice (36) and also the observations of the other authors (37-39) we did not observe the differences in the rate of neuropathy between patients receiving bortezomib SC and IV. Grade 3/4 non-hematological complications were rare, with neutropenia observed only in $2.5 \%$ of patients similarly to the Italian retrospective analysis by Cavo et al (23) (2\%) and much lower than in the IFM2013-04 trial 1(8.9\%) (16). The lower incidence of serious adverse events might result from earlier decisions on dose reductions or treatment discontinuation in clinical practice than in clinical trials.

In the majority of MM patients undergoing subsequent stem cell mobilization, one apheresis procedure was sufficient to obtain HSC for two transplants. These results confirm that VTD does not negatively impact HSC collection $(16,22,40)$, even given at the higher number of cycles. In contrast, lenalidomide was reported to adversely impair HSC harvest (41-43), though in the more recent retrospective analysis a sufficient number of stem cells was obtained in MM patients treated with lenalidomide-based regimens (44). In our study, cytosine arabinoside that is often used in Polish transplantation centers allowed to achieve higher numbers of HSC compared to cyclophosphamide, confirming the previous data from retrospective analysis of the Polish Lymphoma Research Group (45). Efficient method of HSC mobilization was also G-CSF alone.

After HDT/ASCT there was an improvement in the depth of response with increase rate of $\geq V G P R, C R$ and $s C R$. As it was demonstrated, achievement of $\geq$ VGPR after transplant has a significant effect for prolongation of PFS and OS (46-49). Tandem ASCT allowed for a further increase of CR, similarly to the previous reports $(50,51)$. Neutrophils and platelets engraftment after ASCT was not impaired in patients treated with VCT compared to the other protocols used in induction $(16,22,52)$.

This study has some limitations, such as the differences in the regimen received by patients ( 21 vs. 28 day cycles), thalidomide/dexamethasone dosing, bortezomib routes administration and limited availability of cytogenetics/FISH tests that result from retrospective data analysis.

One the other hand, these diversities reflect the management of the MM patients in routine clinical practice. In spite of VTD extensive evaluation in randomized comparative late phase clinical trials, in 'real world' MM patient populations are much more divergent (53) that affects the clinical outcomes. This real-world analysis is very important from the clinical point of view, the more so it is the first of its kind in Poland.

In summary, VTD as induction regimen in MM patients eligible to ASCT allowed high rates of response to be achieved in routine clinical practice, though more cycles were needed to obtain results like those in clinical trials. These data suggest that in some patients it would be useful to give more than 4 cycles to optimize the quality of response, especially as the treatment with the median of 6 cycles was well tolerated and had no negative impact on stem cell collection or hematopoiesis reconstitution after ASCT. However, attention should be paid to more adequate neuropathy assessment and appropriate bortezomib/thalidomide dose reduction. 


\section{Acknowledgements}

Not applicable.

\section{Funding}

The present study was funded by a research grant obtained from the Medical University of Lublin, Poland (grant no. DS174).

\section{Availability of data and materials}

All data generated or analyzed during the present study are included in this published article.

\section{Authors' contributions}

IH, JM and AD designed the study, IH and JM analyzed the data. DJ and AS performed the statistical analysis. IH and JM performed the literature search and data extraction. IH and JM wrote the manuscript. IH, JM, DJ, AJ, GC, KPG, LUZ, MS, ADS, AŚ, AK, NG, MRa, AW, AP, AG, DD, TK, MRo, AWG, JDS, BP, AP, MDD, AS and AD. contributed to the acquisition and interpretation of the clinical data. IH, JM and AS reanalyzed the data and manuscript after revision. AD critically revised the article for intellectual content. All authors read the manuscript and approved the final version.

\section{Ethics approval and consent to participate}

Not applicable.

\section{Patient consent for publication}

Not applicable.

\section{Competing interests}

The authors declare that they have no competing interests.

\section{References}

1. Moreau P, San Miguel J, Sonneveld P, Mateos MV, Zamagni E, Avet-Loiseau H, Hajek R, Dimopoulos MA, Ludwig H, Einsele H, et al: Multiple myeloma: ESMO Clinical practice guidelines for diagnosis, treatment and follow-up. Ann Oncol 28 (Suppl 4): iv52-iv61, 2017.

2. Munshi NC, Anderson KC, Bergsagel PL, Shaughnessy J, Palumbo A, Durie B, Fonseca R, Stewart AK, Harousseau JL, Dimopoulos M, et al: Consensus recommendations for risk stratification in multiple myeloma: Report of the International Myeloma workshop consensus panel 2. Blood 117: 4696-4700, 2011.

3. Anderson KC: The 39th David A. Karnofsky Lecture: Bench-to-bedside translation of targeted therapies in multiple myeloma. J Clin Oncol 30: 445-452, 2012.

4. Palumbo A and Anderson K: Multiple myeloma. N Engl J Med 364: 1046-1060, 2011.

5. Kumar SK, Callander NS, Alsina M, Atanackovic D, Biermann JS, Chandler JC, Costello C, Faiman M, Fung HC, Gasparetto C, et al: Multiple myeloma, version 3.2017, NCCN clinical practice guidelines in oncology. J Natl Compr Canc Netw 15: 230-269, 2017.

6. Mateos MV, Ocio EM, Paiva B, Rosiñol L, Martínez-López J, Bladé J, Lahuerta JJ, García-Sanz R and San Miguel JF: Treatment for patients with newly diagnosed multiple myeloma in 2015 . Blood Rev 29: 387-403, 2015.

7. Rajkumar SV: Multiple myeloma: 2016 update on diagnosis, risk-stratification, and management. Am J Hematol 91: 719-734, 2016.
8. Dmoszynska A, Walter-Croneck A, Usnarska-Zubkiewicz L, Stella-Holowiecka B, Walewski J, Charliński G, Jedrzejczak W, Wiater E, Lech-Maranda E, Dytfeld D, et al: Recommendations of polish myeloma group concerning diagnosis and therapy of multiple myeloma and other plasmacytic dyscrasias for 2015. Acta Hematol Pol 46: 159-211, 2015.

9. Kyle RA and Rajkumar SV: Criteria for diagnosis, staging, risk stratification and response assessment of multiple myeloma. Leukemia 23: 3-9, 2009.

10. Greipp PR, San Miguel J, Durie BG, Crowley JJ, Barlogie B, Bladé J, Boccadoro M, Child JA, Avet-Loiseau H, Kyle RA, et al: International staging system for multiple myeloma. J Clin Oncol 23: 3412-3420, 2005.

11. Palumbo A, Avet-Loiseau H, Oliva S, Lokhorst HM, Goldschmidt H, Rosinol L, Richardson P, Caltagirone S, Lahuerta JJ, Facon T, et al: Revised international staging system for multiple myeloma: A report from international myeloma working group. J Clin Oncol 33: 2863-2869, 2015.

12. Voorhees PM and Usmani SZ: The role of high-dose melphalan and autologous stem cell transplant in the rapidly evolving era of modern multiple myeloma therapy. Clin Adv Hematol Oncol 14: 719-728, 2016.

13. Hari P: Recent advances in understanding multiple myeloma. Hematol Oncol Stem Cell Ther 10: 267-271, 2017.

14. Cavo M, Tacchetti P, Patriarca F, Petrucci MT, Pantani L, Galli M, Di Raimondo F, Crippa C, Zamagni E, Palumbo A, et al: Bortezomib with thalidomide plus dexamethasone compared with thalidomide plus dexamethasone as induction therapy before, and consolidation therapy after, double autologous stem-cell transplantation in newly diagnosed multiple myeloma: A randomised phase 3 study. Lancet 376: 2075-2085, 2010.

15. Moreau P, Avet-Loiseau H, Facon T, Attal M, Tiab M, Hulin C, Doyen C, Garderet L, Randriamalala E, Araujo C, et al: Bortezomib plus dexamethasone versus reduced-dose bortezomib, thalidomide plus dexamethasone as induction treatment before autologous stem cell transplantation in newly diagnosed multiple myeloma. Blood 118: 5752-5758, 2011.

16. Moreau P, Hulin C, Macro M, Caillot D, Chaleteix C, Roussel M, Garderet L, Royer B, Brechignac S, Tiab M, et al: VTD is superior to VCD prior to intensive therapy in multiple myeloma: Results of the prospective IFM2013-04 trial. Blood 127: 2569-2574, 2016.

17. Durie BG, Hoering A, Abidi MH, Rajkumar SV, Epstein J, Kahanic SP, Thakuri M, Reu F, Reynolds CM, Sexton R, et al: Bortezomib with lenalidomide and dexamethasone versus lenalidomide and dexamethasone alone in patients with newly diagnosed myeloma without intent for immediate autologous stem-cell transplant (SWOG S0777): A randomised, open-label, phase 3 trial. Lancet 389: 519-527, 2017.

18. Wang A, Duan Q, Liu X, Ding K, Han Y, Zhu W, Cai X, Wu J and Sun Z: (Bortezomib plus lenalidomide/thalidomide)-vs. (bortezomib or lenalidomide/thalidomide)-containing regimens as induction therapy in newly diagnosed multiple myeloma: A meta-analysis of randomized controlled trials. Ann Hematol 91: 1779-1784, 2012.

19. Landgren $\mathrm{O}$ and Iskander $\mathrm{K}$ : Modern multiple myeloma therapy: Deep, sustained treatment response and good clinical outcomes. J Intern Med 281: 365-382, 2017.

20. Huang H, Zhou L, Peng L, Fu W, Zhang C and Hou J: Bortezomib-thalidomide-based regimens improved clinical outcomes without increasing toxicity as induction treatment for untreated multiple myeloma: A meta-analysis of phase III randomized controlled trials. Leuk Res 38: 1048-1054, 2014.

21. Ludwig H, Viterbo L, Greil R, Masszi T, Spicka I, Shpilberg O, Hajek R, Dmoszynska A,Paiva B, Vidriales MB, et al: Randomized phase II study of bortezomib, thalidomide, and dexamethasone with or without cyclophosphamide as induction therapy in previously untreated multiple myeloma. J Clin Oncol 31: 247-255, 2013.

22. Rosinol L, Oriol A, Teruel AI, Hernández D, López-Jiménez J, de la Rubia J, Granell M, Besalduch J, Palomera L, González Y, et al: Superiority of bortezomib, thalidomide, and dexamethasone (VTD) as induction pretransplantation therapy in multiple myeloma: A randomized phase 3 PETHEMA/GEM study. Blood 120: 1589-1596, 2012.

23. Cavo M, Pantani L, Pezzi A, Petrucci MT, Patriarca F, Di Raimondo F, Marzocchi G, Galli M, Montefusco V, Zamagni E, et al: Bortezomib-thalidomide-dexamethasone (VTD) is superior to bortezomib-cyclophosphamide-dexamethasone (VCD) as induction therapy prior to autologous stem cell transplantation in multiple myeloma. Leukemia 29: 2429-2431, 2015. 
24. Avet-Loisseau $H$, Leleu X Roussel M, Moreau P, Guerin-Charbonnel C, Caillot D, Marit G, Benboubker L, Voillat L, Mathiot $\mathrm{C}$, et al: Bortezomib plus dexamethasone induction improves outcome of patients with $\mathrm{t}(4 ; 14)$ myeloma but not outcome of patients with del(17p). J Clin Oncol 28: 4630-4634, 2010.

25. Mateos MV, Gutierrez NC, Martín-Ramos ML, Paiva B, Montalbán MA, Oriol A, Martínez-López J, Teruel AI, Bengoechea E, Martín A, et al: Outcome according to cytogenetic abnormalities and DNA ploidy in myeloma patients receiving short induction with weekly bortezomib followed by maintenance. Blood 118: 4547-4553, 2011

26. Shaughnessy JD, Zhou Y, Haessler J, van Rhee F, Anaissie E, Nair B, Waheed S, Alsayed Y, Epstein J, Crowley J and Barlogie B: TP53 deletion is not an adverse feature in multiple myeloma treated with total therapy 3. Br J Haematol 147: 347-351, 2009.

27. An G, Acharya C, Deng S, Yi S, Xu Y, Qin X, Sui W, Li Z, Shi L, Zang M, et al: Cytogenetic and clinical marks for defining high-risk myeloma in the context of bortezomib treatment. Exp Hematol 43: 168-176.e2, 2015

28. Neben K, Lokhorst HM, Jauch A, Bertsch U, Hielscher T, van derHolt B,SalwenderH,Blau IW, Weisel K,PfreundschuhM, et al: Administration of bortezomib before and after autologous stem cell transplantation improves outcome in multiple myeloma patients with deletion 17p. Blood 119: 940-948, 2012.

29. Sonneveld P, Salwender H, Van Der Holt B, El Jarari L, Bertsch U, Blau LW,Zweegman S, Weisel KC, VellengaE,Pfreundschuh M, et al: Bortezomib induction and maintenance in patients with newly diagnosed multiple myeloma: Long-term follow-up of the HOVON-65/GMMG-HD4 trial. Blood 126: 27, 2015.

30. Moreau P, Attal M, Pégourié B, Planche L, Hulin C, Facon T, Stoppa AM, Fuzibet JG, Grosbois B, Doyen C, et al: Achievement of VGPR to induction therapy is an important prognostic factor for longer PFS in the IFM2005-01 trial. Blood 117: 3041-3044, 2011.

31. Rajkumar SV, Jacobus S, Callander NS, Fonseca R, Vesole DH, Williams ME, Abonour R, Siegel DS, Katz M and Greipp PR; Eastern Cooperative Oncology Group: Lenalidomide plus high-dose dexamethasone versus lenalidomide plus low-dose dexamethasone as initial therapy for newly diagnosed multiple myeloma: An open-label randomised controlled trial. Lancet Oncol 11: 29-37, 2010.

32. Richardson PG, Weller E, Lonial E, Jakubowiak AJ, Jagannath S, Raje NS, Avigan DE, Xie W, Ghobrial IM, Schlossman RL, et al: Lenalidomide, bortezomib, and dexamethasone combination therapy in patients with newly diagnosed multiple myeloma. Blood 116: 679-686, 2010.

33. Tacchetti P, Terragna C, Galli M, Zamagni E, Petrucci MT, Pezzi A, Montefusco V, Martello M, Tosi P, Baldini L, et al Bortezomib- and thalidomide-induced peripheral neuropathy in multiple myeloma: Clinical and molecular analyses of a phase 3 study. Am J Hematol 89: 1085-1091, 2014.

34. LeibaM,KedmiM,DuekA,FreidmanT,WeissM,LeibaR,NaglerA and Avigdor A: Bortezomib-cyclophosphamide-dexamethasone (VCD) versus bortezomib-thalidomide-dexamethasone (VTD)-based regimens as induction therapies in newly diagnosed transplant eligible patients with multiple myeloma: A meta-analysis. Br J Haematol 166: 702-710, 2014.

35. Moreau P, Pylypenko H, Grosicki S, Karamanesht I, Leleu X, Grishunina M, Rekhtman G, Masliak Z, Robak T, Shubina A, et al: Subcutaneous versus intravenous administration of bortezomib in patients with relapsed multiple myeloma: A randomised, phase 3, non-inferiority study. Lancet Oncol 12: 431-440, 2011.

36. Hus I, Walter-Croneck A, Masternak A, Jurczyszyn A, Usnarska-Zubkiewicz L, Bołkun $Ł$, Druzd-Sitek A, Rymko M, Łettowska J, Lech-Marańda E, et al: Real-life experience with bortezomib-based regimens in elderly patients with newly diagnosed multiple myeloma and comorbidities: A Polish retrospective multicenter study. Pol Arch Intern Med 127: 765-774, 2017.

37. Goldschmidt H, Moreau P, Ludwig H, Niesvizky R, Chng WJ Joshua D, Weisel K, Spencer A, Orlowski RZ, Feng S, et al: Carfilzomib-dexamethasone versus subcutaneous or intravenous bortezomib in relapsed or refractory multiple myeloma: Secondary analysis of the phase 3 ENDEAVOR study. Leuk Lymphoma 59: 1364-1374, 2018.

38. Merz M, Salwender H, Haenel M, Mai EK, Bertsch U, Kunz C, Hielscher T, Blau IW, Scheid C, Hose D, et al: Peripheral neuropathy associated with subcutaneous or intravenous bortezomib in patients with newly diagnosed myeloma treated within the GMMG MM5 phase III trial. Haematologica 101: e485-e 487, 2016.
39. Minarik J, Pavlicek P, Pour L, Pika T, Maisnar V, Spicka I, Jarkovsky J, Krejci M, Bacovsky J, Radocha J, et al: Subcutaneous bortezomib in multiple myeloma patients induces similar therapeutic response rates as intravenous application but it does not reduce the incidence of peripheral neuropathy. PLoS One 10: e0123866, 2015.

40. Brioli A, Perrone G, Patriarca F, Pezzi A, Nobile F, Ballerini F, Motta MR, Ronconi S, Tacchetti P, Catalano L, et al: Successful mobilization of PBSCs predicts favorable outcomes in multiple myeloma patients treated with novel agents and autologous transplantation. Bone Marrow Transplant 50: 673-678, 2015.

41. Kumar S, Dispenzieri A, Lacy MQ, Hayman SR, Buadi FK, Gastineau DA, Litzow MR, Fonseca R, Roy V, Rajkumar SV and Gertz MA: Impact of lenalidomide therapy on stem cell mobilization and engraftment post-peripheral blood stem cell transplantation in patients with newly diagnosed myeloma. Leukemia 21: 2035-2042, 2007.

42. Paripati H, Stewart AK, Cabou S, Dueck A, Zepeda VJ, Pirooz N, Ehlenbeck C, Reeder C, Slack J, Leis JF, et al: Compromised stem cell mobilization following induction therapy with lenalidomide in myeloma. Leukemia 22: 1282-1284, 2008.

43. Kumar S, Giralt S, Stadtmauer EA, Harousseau JL, Palumbo A, Bensinger W, Comenzo RL, Lentzsch S, Munshi N, Niesvizky R, et al: Mobilization in myeloma revisited: IMWG consensus perspectives on stem cell collection following initial therapy with thalidomide-, lenalidomide-, or bortezomib-containing regimens. Blood 114: 1729-1735, 2009.

44. Sinha S, Gertz MA, Lacy MQ, Dispenzieri A, Hayman SR, Buadi FK, Dingli D, Micallef IN, Hogan WJ, Gastineau DA, et al: Majority of patients receiving initial therapy with lenalidomide-based regimens can be successfully mobilized with appropriate mobilization strategies. Leukemia 26: 1119-1122, 2012.

45. Giebel S, Sadus-Wojciechowska M, Halaburda K, Drozd-Sokolowska J, Wierzbowska A, Najda J, Mendrek W, Sobczyk-Kruszelnicka M, Nowicki M, Holowiecki J and Czerw T: Increased efficacy of intermediate-dose cytarabine + G-CSF compared to DHAP + G-CSF for stem cell mobilization in patients with lymphoma: An analysis by the polish lymphoma research group. Ann Hematol 95: 263-269, 2016.

46. Harousseau JL, Attal M and Avet-Loiseau H: The role of complete response in multiple myeloma. Blood 114: 3139-3146, 2009.

47. Child JA, Morgan GJ, Davies FE, Owen RG, Bell SE, Hawkins K, Brown J, Drayson MT and Selby PJ; Medical Research Council Adult Leukaemia Working Party: High-dose chemotherapy with hematopoietic stem cell rescue for multiple myeloma. N Engl J Med 348: 1875-1883, 2003.

48. Wang M, Delasalle K, Feng L, Thomas S, Giralt S, Qazilbash M, Handy B, Lee JJ and Alexanian R: CR represents an early index of potential long survival in multiple myeloma. Bone Marrow Transplant 45: 498-504, 2010.

49. Chanan-Kahn A and Giralt S: Importance of achieving a complete response in multiple myeloma, and the impact of novel agents. J Clin Oncol 28: 2612-2624, 2010.

50. Attal M, Harousseau JL, Facon T, Guilhot F, Doyen C, Fuzibet JG, Monconduit M, Hulin C, Caillot D, Bouabdallah R, et al: Single versus double autologous stem-cell transplantation for multiple myeloma. N Engl J Med 349: 2495-2502, 2003.

51. Cavo M, Tosi P, Zamagni E, Cellini C, Tacchetti $\mathrm{P}$ Patriarca F, Di Raimondo F, Volpe E, Ronconi S, Cangini D, et al: Prospective, randomized study of single compared with double autologous stem-cell transplantation for multiple myeloma: Bologna 96 clinical study. J Clin Oncol 25: 2434-2441, 2007.

52. Benyamini N, Avivi I, Dann EJ, Zuckerman T, Lavi N and Katz T: Comparison of engraftment following different stem cell mobilization modalities in patients with multiple myeloma treated with a uniform induction regimen containing bortezomib, cyclophosphamide and dexamethasone. Ann Hematol 96: 461-467, 2017.

53. Blimark CH, Turesson I, Genall A, Ahlberg L, Björkstrand B, Carlson K, Forsberg K, Juliusson G, Linder O, Mellqvist UH, et al: Outcome and survival of myeloma patients diagnosed 2008-2015. Real-world data on 4904 patients from the Swedish Myeloma Registry. Haematologica 103: 506-513, 2018.

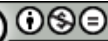

This work is licensed under a Creative Commons Attribution-NonCommercial-NoDerivatives 4.0 International (CC BY-NC-ND 4.0) License. 